\title{
Strategies and Partnerships Toward Prevention of Healthcare-Associated Venous Thromboembolism
}

\author{
Michele G. Beckman, $\mathrm{MPH}^{1 *}$, Karon Abe, $\mathrm{PhD}^{1}$, Kelly Barnes, $\mathrm{MS}^{2}$, Barbara Bartman, MD, MPH${ }^{3}$, \\ P. Jeffrey Brady, MD, MPH${ }^{3}$, W. Craig Hooper, $\mathrm{PhD}^{1}$
}

${ }^{1}$ Division of Blood Disorders, Centers for Disease Control and Prevention, Atlanta, Georgia; '2Joint Commission Center for Transforming Healthcare, Oakbrook Terrace, Illinois; ${ }^{3}$ Agency for Healthcare Research and Quality, Rockville, Maryland.

Venous thromboembolism (VTE), blood clots occurring as deep vein thrombosis, pulmonary embolism, or both, is an important and growing public health issue. The precise number of people affected by VTE is unknown; however, estimates suggest that up to 900,000 events resulting in as many as 100,000 premature deaths occur in the United States yearly with healthcare costs as high as $\$ 10$ billion. ${ }^{1-3}$ Although anyone can develop VTE, research has shown that half of VTE events occurring in the outpatient setting are directly linked to a recent hospitalization or surgery. ${ }^{4}$ In patients with cancer, VTE is a leading cause of death after the cancer itself. ${ }^{5,6}$ Fortunately, many of these healthcare-associated VTE (HA-VTE) cases can be prevented. Recent analyses have shown that as many as $70 \%$ of HA-VTE cases are preventable through appropriate prophylaxis, ${ }^{7-9}$ yet reports suggest that fewer than half of hospital patients receive VTE prophylaxis in accordance with accepted evidence-based guidelines. ${ }^{10}$ Appropriate prevention of HA-VTE can result in a significant reduction in overall VTE occurrence, thereby decreasing healthcare burden and unnecessary deaths.

In November 2015, the Centers for Disease Control and Prevention (CDC) released the HealthcareAssociated VTE Prevention Challenge (http://www.cdc. gov/ncbddd/dvt/ha-vte-challenge.html) to identify, highlight, and reward hospitals, managed care organizations, and hospital networks that implemented innovative, effective, and sustainable strategies to prevent HA-VTE.

This issue of the Journal of Hospital Medicine showcases the initiatives of several of the CDC's HA-VTE prevention champions. These champions range from a small community hospital to some of the country's largest health systems, and they represent both rural and urban areas. Together they cared for more than 450,000 patients admitted to hospitals across the United States in 2014.

*Address for correspondence and reprint requests: Michele G. Beckman, Division of Blood Disorders, Centers for Disease Control and Prevention, 4770 Buford Highway, MS E-64, Chamblee, GA 30341; Telephone: 404-498-6474; Fax: 770-488-0068;

E-mail: mbeckman@cdc.gov

Additional Supporting Information may be found in the online version of this article.

Received: June 16, 2016; Revised: August 26, 2016; Accepted: September 11, 2016

2016 Society of Hospital Medicine DOI 10.1002/jhm.2659

Published online in Wiley Online Library (Wileyonlinelibrary.com).
They were able to improve VTE prevention within their institutions and organizations by implementing successful and sustainable VTE prevention strategies such as engaging teams of different healthcare experts to support and promote prevention activities, informing patients and providers about the need for and benefits of VTE prevention, and using technology (such as electronic risk assessment and clinical decision support tools and alerts) to ensure that all patients are assessed for their risk for VTE and bleeding. These tools also help ensure that patients, when appropriate, are provided with and use appropriate prevention measures for their level of risk. Moreover, they provided real-time feedback, scorecards, and dashboards for providers and organizations to monitor performance and identify areas for improvement.

The CDC and the Agency for Healthcare Research and Quality (AHRQ) are partnering to disseminate and promote these best practices. In addition to this challenge, the CDC, AHRQ and the Joint Commission Center for Transforming Healthcare are working on activities and programs dedicated to improving prevention of HA-VTE. These are summarized below.

\section{AGENCY FOR HEALTHCARE RESEARCH AND QUALITY}

The AHRQ works to reduce the incidence of VTE among patients at risk of developing the condition by conducting research and providing support for quality-improvement efforts. In 2008, AHRQ published an evidence-based resource for prevention of VTE based on quality-improvement principles that were successfully applied by the University of California, San Diego Medical Center, and Emory University Hospitals in an AHRQ-funded research project. This resource, Preventing Hospital-Acquired Venous Thromboembolism: A Guide for Effective Quality Improvement, was updated in 2014 and is available at http://www.ahrq. gov/professionals/quality-patient-safety/patient-safetyresources/resources/vtguide/index.html. The guide is intended to assist quality-improvement practitioners who wish to improve prevention of HA-VTE in their own organizations.

Since its original release, hospital quality-improvement teams have used the guide to close the gap between available evidence about how to prevent VTE and successful implementation of that knowledge so that hospitals can improve care as effectively and efficiently as possible. The 
guide includes examples of risk assessment methodologies and evaluation metrics along with other key elements that help front-line providers establish or enhance VTE prevention programs.

AHRQ maintains an active patient safety program that spans a wide range of patient safety problems including VTE that is aimed at making healthcare safer. The AHRQ works with many different healthcare stakeholders to (1) better understand threats to patient safety and (2) develop and refine strategies that put this knowledge to work to prevent patient harm. For example, the AHRQ provides extramural research grants to investigators studying new methods of identifying patients at risk for VTE and ways to improve VTE prophylaxis in hospital patients undergoing medical treatment and surgical procedures.

In addition to a collaborative partnership with the CDC on the 2015 Healthcare-Associated VTE Prevention Challenge, the AHRQ also partners with other federal agencies and is an active contributor to the Department of Health and Human Services National Action Plan for Adverse Drug Event Prevention. The plan outlines opportunities to advance patient safety through the prevention of adverse drug events and the promotion of medication safety among 3 drug classes including anticoagulants, a key component for VTE prevention. Patients and their families are critically important partners for improving patient safety, and the significant potential for their impact is represented by the AHRQ tools and resources such as an information video and guide for patients about the safe use of blood thinners (http://www.ahrq.gov/patients-consumers/diagnosis-treatment/treatments/btpills/btpills.html).

\section{THE JOINT COMMISSION CENTER FOR TRANSFORMING HEALTHCARE}

The Joint Commission Center for Transforming Healthcare commenced the Preventing Venous Thromboembolism project in October 2014, with 5 participating hospitals and health centers in collaboration with the CDC. The hospitals and health centers participating on this project include: Cleveland Clinic, The Johns Hopkins Hospital, Kaiser Permanente South Bay Medical Center, Massachusetts General Hospital, and Texas Health Resources.

Often, VTE risk factors are not consistently assessed across all hospital patients, and there is much variation to the selection of appropriate mechanical and/or pharmacological prophylaxis. In addition, the current accepted guidelines are not implemented consistently across hospitals. VTE rates can be reduced with accurate risk assessment and appropriate utilization of pharmacological and/or mechanical prophylaxis. However, there are multiple barriers to consistent, successful implementation of preventative measures. During the first year of this project, the participants utilized Robust Process Improvement, a fact-based, systematic, and data-driven problem-solving methodology that incorporates tools and concepts from Lean, Six Sigma, and change management, to assist them in identifying the root causes and barriers to preventing VTE in at-risk patients.

Aggregate preliminary findings show that some of the contributing factors to VTE prevention included issues with staff attitudes and beliefs, staff and patient education, risk assessments, order sets, ineffective mechanical and pharmacological prophylaxis, and patient refusal. The participating organizations are currently developing and implementing solutions targeted to their specific root causes. The solutions will be tested, validated, and then spread to other healthcare organizations. Project findings are tentatively scheduled for release in 2017.

The Joint Commission has been committed to preventing VTE for over a decade, beginning with the development of the VTE measures, which were the result of the National Consensus Standards for the Prevention and Care of Deep Vein Thrombosis project with the National Quality Forum that formally began in January 2005. In addition, The Joint Commission and Joint Commission Resources have also aimed to reduce harm and improve the quality of care for VTE patients through the Hospital Engagement Network collaborative, publications related to improving VTE patient safety, and research on improving transitions of care and discharge instructions for patients with VTE.

\section{CENTERS FOR DISEASE CONTROL AND PREVENTION}

The CDC's Division of Blood Disorders works to prevent VTE and to reduce sickness and death among those who develop VTE. The CDC's primary VTE activities focus on developing improved methods and innovative tools for monitoring and understanding VTE occurrence and the effectiveness of VTE prevention activities, conducting epidemiologic studies on the causes and outcomes of VTE and its complications, and working internally and with partners to develop and promote education and awareness materials to inform the public and healthcare providers about the importance of knowing VTE risk factors to improve prevention, and the importance of knowing the signs and symptoms of VTE to ensure early and accurate diagnosis and treatment of VTE. Recently, the CDC collaborated with the National Blood Clot Alliance, through their Stop the Clot, Spread the Word Campaign, to develop and promote prevention of VTE among hospitalized patients (https://www. stoptheclot.org/spreadtheword). To learn more about the CDC's VTE programs please visit http://www.cdc. gov/ncbddd/dvt/index.html.

The CDC, AHRQ, and The Joint Commission hope that readers will find the successes of the HA-VTE Prevention Champions' initiatives informative and encouraging. They are examples of how any healthcare setting, from a small hospital to a large healthcare system, can 
implement approaches and tools to improve prevention of HA-VTE.

Disclosures: The findings and conclusions in this report are those of the authors and do not necessarily represent the official position of the Centers for Disease Control and Prevention, the Agency for Healthcare Research and Quality, or the Joint Commission Center for Transforming Healthcare. The authors declare no conflicts of interest.

\section{References}

1. U.S. Department of Health and Human Services, Office of the Surgeon General. The Surgeon General's Call to Action to Prevent Deep Vein Thrombosis and Pulmonary Embolism. Rockville, MD: National Heart, Lung, and Blood Institute; 2008.

2. Beckman MG, Hooper WC, Critchley SE, Ortel TL. Venous thromboembolism: a public health concern. Am J Prev Med. 2010;38(4 suppl): S495-S501.

3. Raskob G, Silverstein R, Bratzler D, Heit J, White R. Surveillance for deep vein thrombosis and pulmonary embolism: recommendations from a national workshop. Am J Prev Med. 2010;38(4 suppl):S502S509.

4. Spencer F, Lessard D, Emery C, Reed G, Goldberg R. Venous thromboembolism in the outpatient setting. Arch Intern Med. 2007;167(14):1471-1475.

5. Connolly GC, Francis CW. Cancer-associated thrombosis. Hematology Am Soc Hematol Educ Program. 2013;2013:684-691.

6. Khorana AA. Cancer-associated thrombosis: updates and controversies. Hematology Am Soc Hematol Educ Program. 2012;2012:626-630.

7. Zeidan AM, Streiff MB, Lau BD, et al. Impact of a venous thromboembolism prophylaxis "smart order set": improved compliance, fewer events. Am J Hematol. 2013;88:545-549.

8. Mitchell JD, Collen JF, Petteys S, Holley AB. A simple reminder system improves venous thromboembolism prophylaxis rates and reduces thrombotic events for hospitalized patients. J Thromb Haemost. 2012;10:236-243.

9. Lau BD, Haut ER. Practices to prevent venous thromboembolism: brief review. BMJ Qual Saf. 2014;23:187-195.

10. Kahn S, Morrison D, Cohen J, et al. Interventions for implementation of thromboprophylaxis in hospitalized medical and surgical patients at risk for venous thromboembolism. Cochrane Database Syst Rev. 2013;7:CD008201. 